\title{
Investigation of deformations of a lithium polymer cell using the Digital Image Correlation Method (DICM)
}

\author{
Szabolcs Szalai ${ }^{2}$, Szabolcs Kocsis Szürke ${ }^{1}$, Dóra Harangozó1, Szabolcs Fischer ${ }^{1}$ \\ ${ }^{1}$ Szechenyi Istvan University, Gyor, Hungary, \\ e-mail: szalaisz@sze.hu \\ e-mail: kocsis.szabolcs@ga.sze.hu \\ e-mail: harangozo.dora@sze.hu \\ e-mail: fischersz@sze.hu
}

\begin{tabular}{l} 
Article Info \\
\hline Article history: \\
Received November 17, 2021 \\
Revised January 27, 2022 \\
Accepted February 8, 2022 \\
\hline
\end{tabular}

\section{Keywords:}

GOM ATOS, GOM ARAMIS,

Battery deformation,

DIC,

DICM.

\section{Corresponding Author:}

Szabolcs Fischer,

Szechenyi Istvan University.

Email: fischersz@sze.hu

\begin{abstract}
This research aims to investigate the adaptability of a measurement system or a process in determining the parameters of batteries. Methods are suggested for different applications, and properties gained by these measurements are specified. Deformations of lithium polymer batteries measured by various methodologies are also analyzed in detail. Changes in the geometry of wornout batteries and the localization of the changes can be better understood by applying the results. The GOM ATOS and the GOM ARAMIS systems were applied to characterize lithium polymer batteries. Discontinuous tests were performed and the battery was discharged to $0 \mathrm{~V}$ and then fully charged for both methods. The advantages and disadvantages and the applicability of the two measurement systems were analyzed in this topic.
\end{abstract}

Copyright (C) 2022 Regional Association for Security and crisis management and European centre for operational research. All rights reserved.

\section{Introduction}

A tremendous demand appeared to produce elements installed in electric vehicles (EV) consuming chemical energy in the last decades. The design and production processes of batteries are also challenged by different needs and application methods (Dunn et al., 2015). Other types of batteries are required to drive vehicles or in entertainment technology (Ehrlich, 2002). However, they have a common feature: a higher energy density is needed to operate reliably in the long run (Slattery, Dunn \& Kendall, 2021). Therefore, the energy management, efficient analysis, and prediction of the physical condition of batteries used in electric vehicles is a priority in favor of economic usage (Rezvanizaniani et al., 2014; Fotouhi et al., 2016; Zheng et al., 2016; Zhu, Wierzbicki \& Li, 2018; How et al., 2019; Wang et al., 2020). The authors tested lithium polymer batteries applied to vehicles and entertaining technology products to determine deformities.

In the everyday use of lithium polymer batteries, deformations occur. Generally, they swell during charging and contract during discharging (Zhang et al., 2015; Sauerteig et al., 2017). Different solutions are created to detect and measure the geometrical change (Li, 2011; Yufit et al., 2011; Popp et al., 2020; Komsiyska et al., 2021). Tactile methods, distance sensors, and strain gauge stamps are widely used (Rieger et al., 2016). However, these methods can detect the deformation at only a few selected points. This disadvantage can be eliminated by using contactless optical measurement methods, which have already been widely used to 
determine the composition of electrodes and structural properties of batteries (Jones et al., 2014; Leung et al., 2014; Tao et al., 2020).

In the authors' previous publication, they have already measured deformation with the GOM system, focusing on the selection of critical regions of the battery (Kocsis Szürke et al., 2022). The present study examines the two different measurements' advantages, disadvantages, and usability.

Different methods for analyzing deformations and displacements are generally known in literature and practical measurement technology. Strain gauge stamps and inductive displacement sensors are traditional methods that need a lot of preparation and expensive instruments. Digital Image Correlation (DIC) techniques presented in this paper are contactless processes that enable the full-field analysis of the surface deformations and strains with everyday devices with the help of retrospective processes. (Remark: DICM means Digital Image Correlation Method). The location of the failure is not necessary to be known in advance since the results of the measurements concern the whole sample, and their processing is executed subsequently. Digital image correlation as a measurement technique was introduced at a university in South Carolina by a research group in the '80s. In the next three decades, the method was further developed and widespread. Thanks to its many advantages, e.g., simple experimental settings, variability, and widely adjustable resolution in time and space, the DIC technique became a distinguished tool for determining deformations. Nowadays, researchers use this technique willingly, which is presented in Figure 1. The dynamic incline in DIC application can be seen among articles published on the Web of Science between 2000 and 2015. Data of the Google Scholar show a similar tendency (Reu, 2012; Dong \& Pan, 2017).
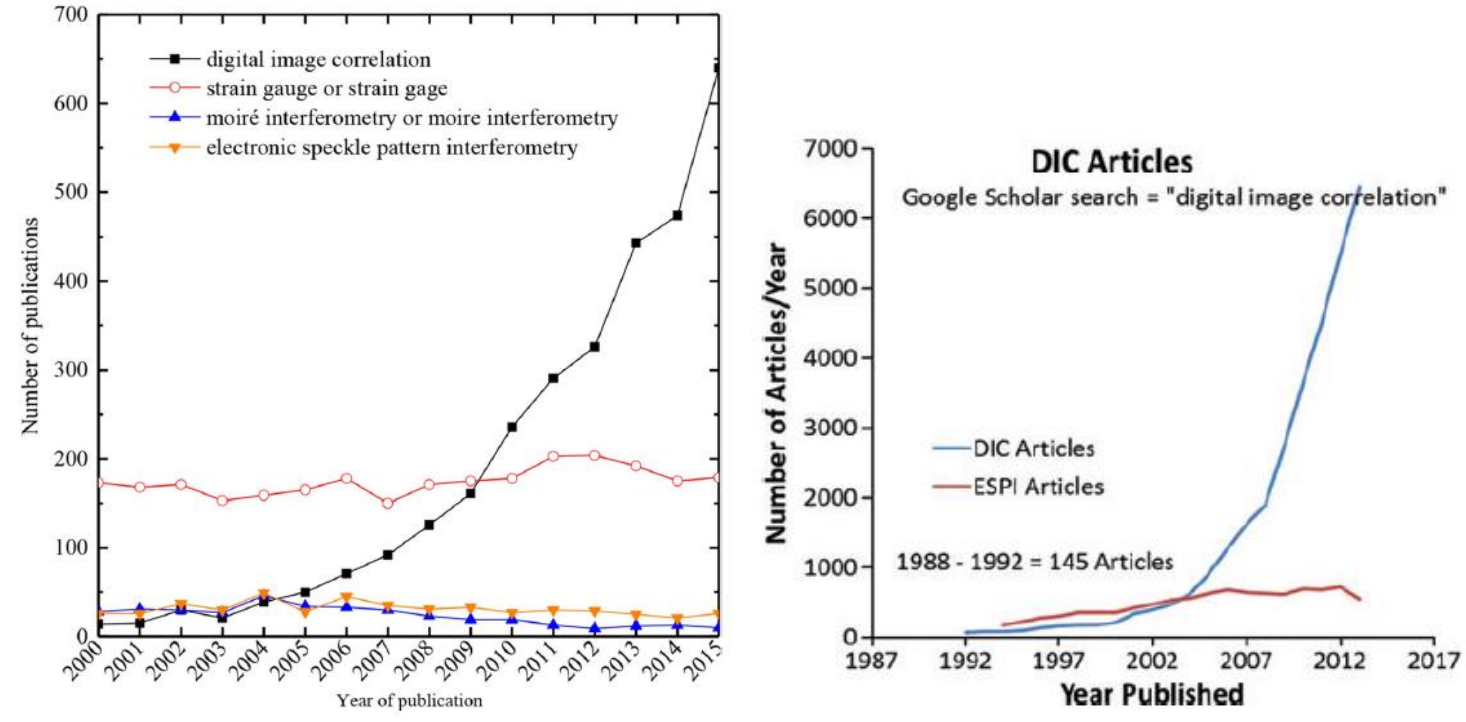

Figure 1. The incline in the application of the DIC technique in scientific publications (Dong \& Pan, 2017)

Based on the above explanation, DIC methods became one of the most critical tools of measurement techniques among experimental engineering and research groups with progressive future applicability. The principle, which is valid either in microscopic (a sample with $\mathrm{mm}$ dimensions) or macroscopic (a sample with $\mathrm{m}$ dimensions) scales, is a significant advantage of DIC. Beyond the widely adaptable scales, the materials to be examined also cover a wide range: the technique is ordinarily used in the analysis of metals, as well as polymers, composites, and biological samples. Not only traditional specimens can be examined, but complex parts, machines, or even parts of buildings, not to mention their usage in biomechanics or medicine (Gualtieri, 2012).

In the last decades parallel to DIC techniques, a more widespread measurement technique called 3D optical surface digitization methods developed dynamically. It is based on photogrammetry. Their intense improvement was supported by the permanent development also observable in the vehicle industry. Traditional measurement techniques could not compete with the more common freeform surfaces or asymmetric parts, which provide the best air resistance. The modern machines based on the principle of surface digitization offer full-field data of the surface, compared to the coordinate measuring machines. The amount of the specimens to be measured is almost unlimited thanks to contactless measurement, which is suitable for samples with dimensions of a few millimeters to more meters (Luhmann et al., 2019; GOM, 2021).

Photogrammetry is contemporary to photography. The first paper-based photo was taken in 1826 , and its applicability in measurement technology was recognized soon. Lussedat, a French official and scientist, and 
Meydenbauer, a German architect, used photos for measurement purposes in 1851 and 1858. The latter used photogrammetry to assess the facade of the dome of Wetzlar (Figure 2.). Thereafter the technique developed parallel to photography and cameras. Nowadays, the most modern hardware and software are available for engineers and scientists (Luhmann et al., 2019).

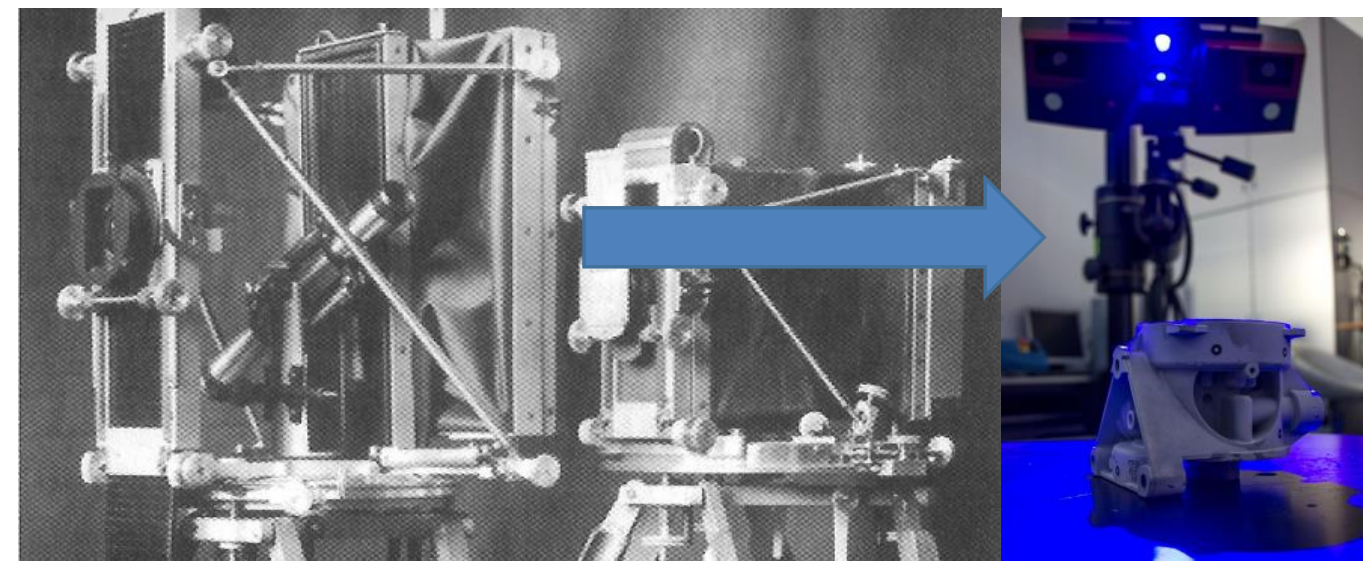

Figure 2. Measuring camera from Meydenbauer (ca. 1890) and the GOM Core (Luhmann et al., 2019; GOM, 2021)

Both two-dimensional three-dimensional field data are measured. Two different measurement techniques have been applied in this study, i.e., structured light (projected blue light with fringes) and Digital Image Correlation (DIC). By using the technique of structured light combined with a calibrated camera model, continuous three-dimensional full-field out-of-plane deformations of plates subjected to impact have been measured. However, this technique does not allow for measurements of strain fields in the test specimen. On the other hand, two-dimensional in-plane displacement and strain fields may be measured by applying DIC on plane specimens.

\section{Materials and methods}

The two measurement methods based on different principles are presented in this section. In addition, a general description and characterization of the process of the measurements and validation are also given.

\subsection{DIC and 3D optical measurement systems}

\section{GOM ATOS}

An industrial 3D scanner with high accuracy, GOM ATOS TripleScan, was utilized during the research. The system's main advantage is the delivery of accurate three-dimensional measurement data to execute industrial processes as precisely as possible and help production processes. ATOS systems are widely used in different industries, e.g., sheet metal parts, tools, casting, blades of rotors, prototypes, injection-molded or cast parts, and tools for injection molding or casting.

Measurement limits are widely variable, which enables the measurements of a finished car but tiny electronic parts also. The device records a dense point cloud or polygon mesh by measuring the whole surface and geometry of the part. It describes precisely the surface of the sample that shows hidden faults, which accelerates and makes production more efficient. An exact pattern of stripes is projected on the surface to be measured, which is recorded simultaneously by two cameras based on the principle of stereo cameras (DIC). Since the cameras and the projector are calibrated, 3D surface points can be determined by triangulation from any two viewpoints of the three devices. The projector emits low-frequency blue light, which helps to eliminate interfering environmental effects. The resolution of the system is $0.01 \mathrm{~mm}$; the accuracy of the measurement depends on the measurement limit. The cameras are measurement technique lenses used in the industry with 8-megapixel resolution. The measurement distance can be altered between 0.49 and 2 meters. According to the measurement limit, the area to be scanned in one step varies between $38 \times 29 \mathrm{~mm}$ and $2000 \times 1500 \mathrm{~mm}$. Generally, small products can be measured with an accuracy of micrometers, while in the case of big parts, this value can be some decades (GOM GmbH, 2011, 2021; GOM, 2021b). 


\section{Principle of the measurement (GOM ATOS)}

The digitization of ATOS 3D scanners is based on the principle of triangulation. The sensor unit projects a stripe pattern of different widths for the object to be measured, and the CCD observes the change of sinusoidal offset phase light intensity reflected by the object. Two cameras record these patterns. The device applies multiple phase offset according to the principle of heterodyne light interference to reach the best sub-pixel accuracy. All the 3D coordinates belonging to camera pixels (up to $4 \mathrm{MP}$ ) are calculated in seconds automatically and with high accuracy by the computer based on transformation equations of optical imaging. To digitize the whole surface of an object, more independent measurements from different views are needed. The ATOS system applies a unique technique to recognize reference points to combine data collected from different perspectives. While measuring the object, the system simultaneously identifies the reference points placed on the thing or the surrounding geometry before the measurement. All the results are automatically combined in a common global coordinate system utilizing the reference points. In the case of smaller objects, the reference points can be scanned directly with ATOS. In the case of large things, this can be executed by the TRITOP digital photogrammetric system (NASA, 2014; GOM GmbH, 2011; Bajusz et al., 2019).

It is essential to mention that the measurement system is calibrated by a photogrammetric method, while temperature, geometrical configuration, and distortion parameters of lenses are reconciled.

After scanning, the software calculates a high-resolution polygon mesh for the object's surface, placing small triangles on the curvatures and big triangles on the flat surfaces without decreasing the accuracy of the mesh. As a result, shapes like holes, patterns of holes, grooves, incisions, or edges on the object can be identified by the contrast of the images. Projecting this geometrical information on the polygon mesh, accurate coordinates and dimensions of the sample can be gained.

Black and white images are taken for GOM measurements. The quantity of pixels (pixel resolution) is not the most critical factor in practice. The noise level of the photographs and the optical resolution of the objectives or lenses are most important. The software analyzes the grayscale transitions during the measurement (see Figure 3). The recognition and identification of reference points are crucial because they are used to concatenate. A special ellipse searching algorithm is applied for this task. During ellipse searching, observing the object at a given angle, the target appears as an ellipse (exception: observing from a too sharp edge, the projection distorts the circle shape of the marks). The starting point is approximately in the center of the ellipse. Then the system analyzes along the radius where the grayscale transition occurred, determining the outlines of circles or ellipses, see Figure 4 (Li et al., 2016).

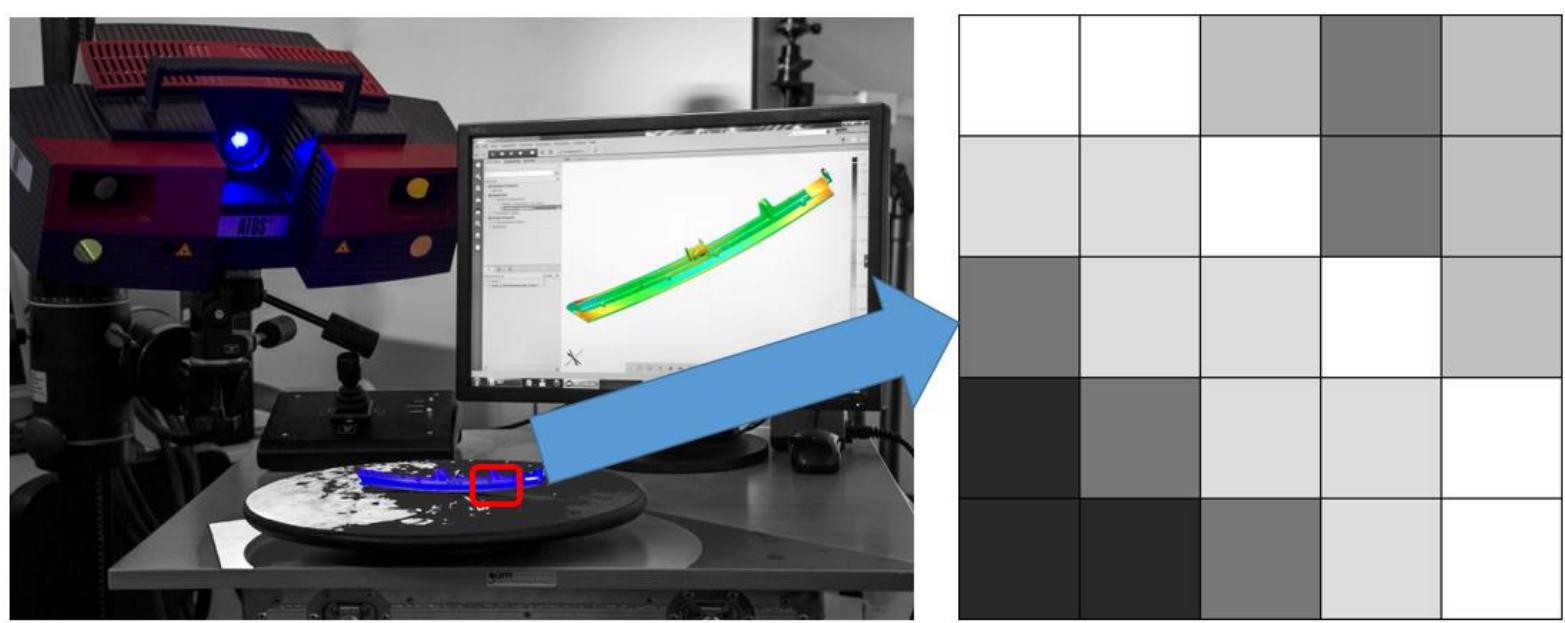

Figure 3. ATOS System records black and white images and detects the grayscale pixel transition 

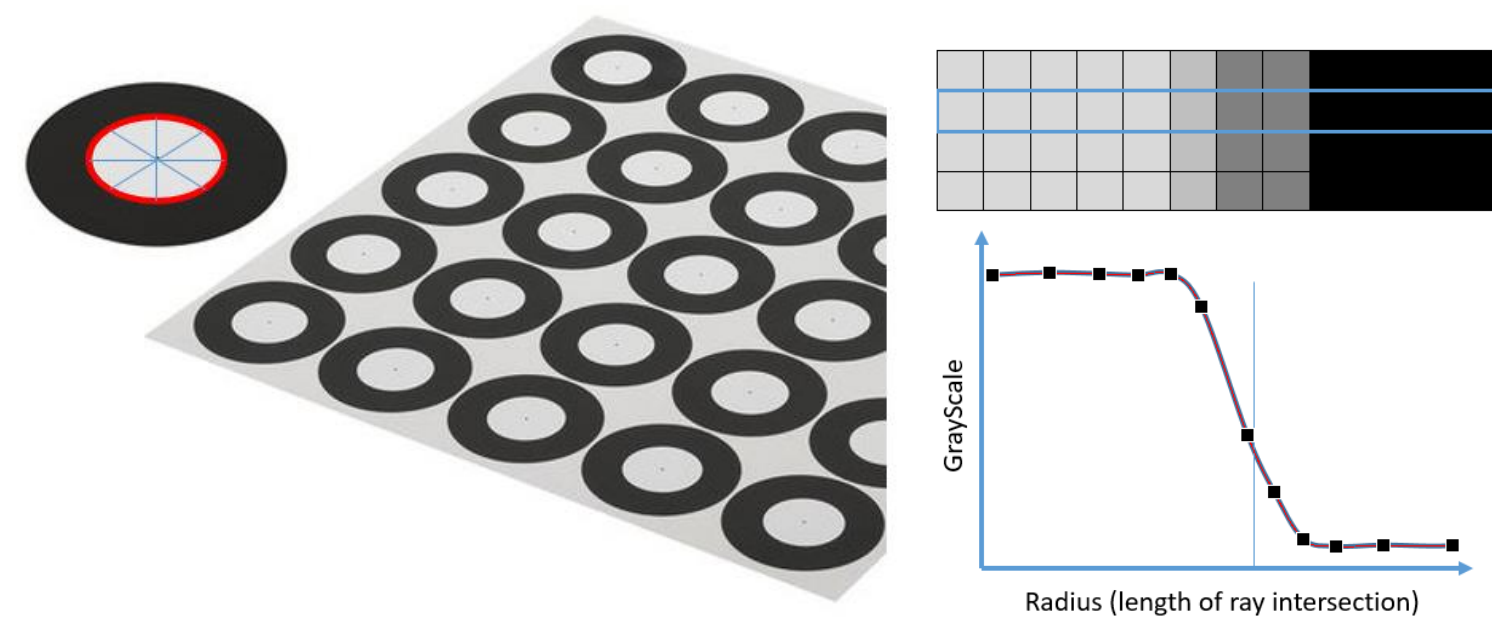

Figure 4. Detection of ellipses and grayscale changes along the ray intersection (GOM, 2021)

GOM applies line or stripe pattern projection during the measurements; thereby, there is no need to move a 'line scanner' that provides permanent resolution surface-based 3D information. Another advantage of the method is the high density of the measurement points on the surface. Still, the increased sensitivity to the projection method and the need for a more complex computational capacity are disadvantages.

Stripe projection (Figure 5) is used to code the object's surface to be measured.

The creation of structured light is the basis for measuring a surface's geometry by a contactless method. In this case, the pattern of the light projected on the surface changes compared to a reference plane used to determine the surface coordinates. The GOM system uses two cameras and a blue light stripe projector (Figure 5). The light pattern is projected to the object's surface to be measured, which is a binary pattern following Gray-code and sinusoidal offset phase light pattern. Gray-code provides unique code while the offset phase enables high local resolution. The advantage of projecting combined light stripes is the stability of the measurement process and the high accuracy of the measurement (Figure 6). To estimate the distance from the reference planes, the geometrical relationships of the camera, the projector, and the coordinate system is required. This is determined during the calibration process while the properties and location (e.g., position and orientation) of the camera are specified (Vagovský, Buranský \& Görög, 2015; Li et al., 2016; Luhmann et al., 2019)

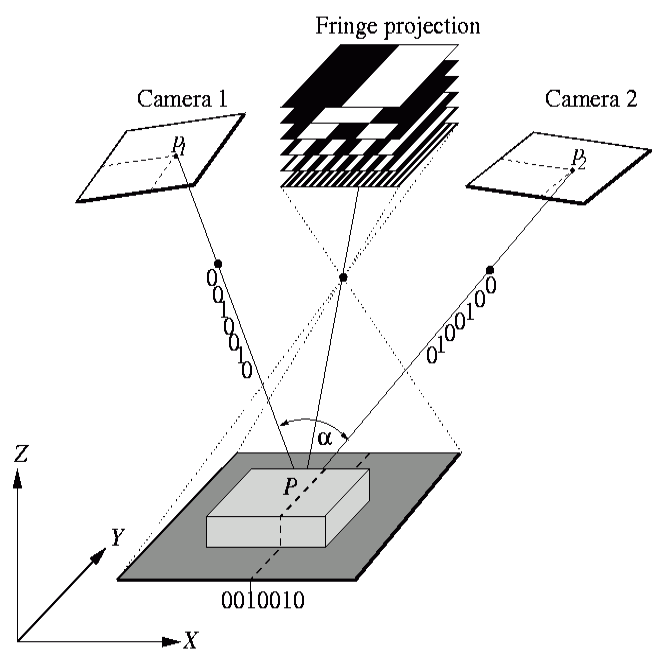

Figure 5. Fringe projection method (GOM, 2021) 


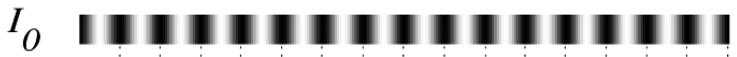

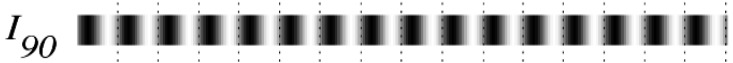 $I_{180} \mathbf{n} \mathbf{n} \mathbf{n} \mathbf{n} \mathbf{n} \mathbf{n} \mathbf{n} \mathbf{n} \mathbf{n} \mathbf{n} \mathbf{n}$

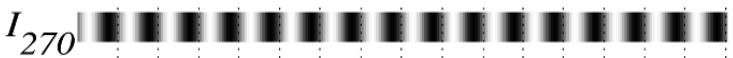
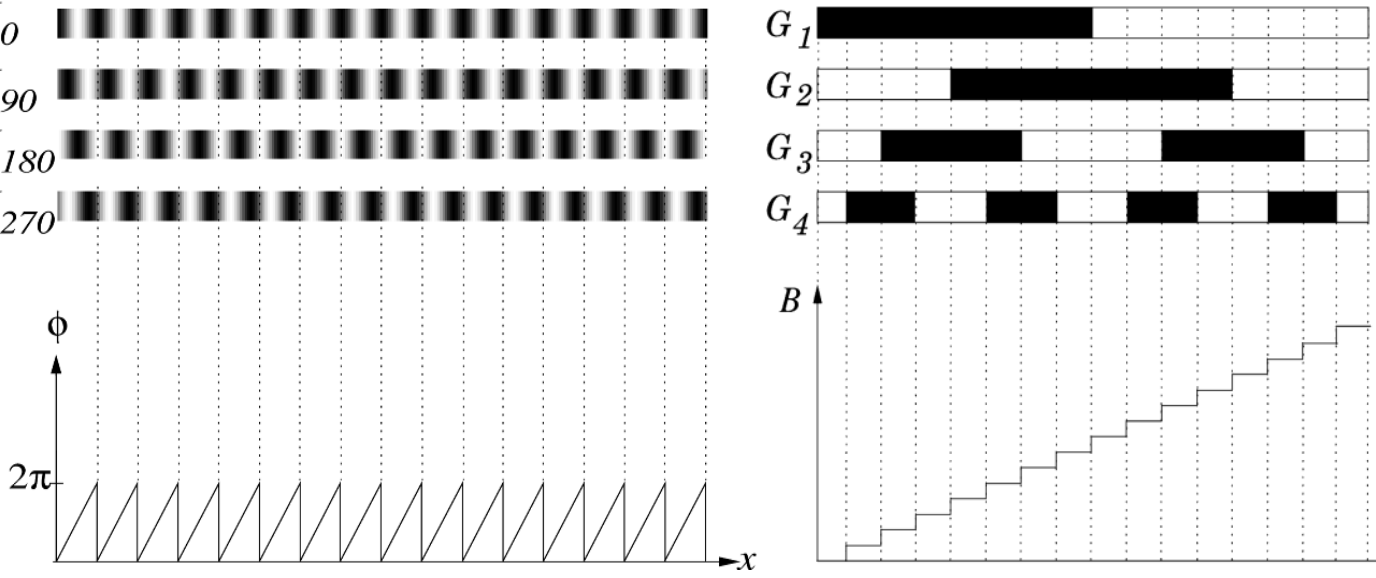

Figure 6. 4-level Phase Shift and 4-level Gray-code (GOM, 2021)

Obviously, a single image is not sufficient to describe a 3D object; therefore taking more photos is needed. The combination of the images is the crucial point of the measurement process. Different surfaces must be merged into one digital object, a so-called point cloud. The coordinate system of the measurement machine and the object have to be reconciled when capturing the images. GOM uses reference points (albeit merging the images by surface specifications is also possible) to merge the different scanning positions using the ellipse method presented above. The condition of spatial transformation is the presence of three common reference points in each image. The advantages of the technique are the stability of the measurement process, the lack of propagation of faults, the ability to measure objects with great dimensions, and the sensor's movement can be detected during measurements. Although the time of preparation can increase significantly (Bajusz et al., 2019; Goda, Lhostis \& Guerlain, 2019; Luhmann et al., 2019).

\section{GOM ARAMIS}

ARAMIS systems are contactless material-independent measurement systems that operate based on digital image correlation. They provide reliable solutions for entire surface and point-based analysis, whether the object's dimensions are a few millimeters or more meters. The sensors of this product group can measure 3D coordinates, 3D displacement, 3D surface deformation dynamically. In addition, characterization of raw material is also possible, e.g., the determination of FLC (forming limit curve) or tensile tests tracked by the DIC technique. The most modern technology enables high local resolution recorded by two 12 MP cameras. Not only deformations on small scales but even effects performing on large extents can be detected. The measurements are helped by variable consoles of the camera and pre-set certified lenses; therefore, the system can be easily and quickly altered depending on the actual area to be measured. Systems designed for industrial use are stable, which means less sensor calibration. The maximum image recording speed of AMAMIS 5M with the highest resolution is 25 fps. Decreasing the width or height of the image, this value can be increased, thus incorrect behavior of parts can be documented in the function of time in detail.

Principle of measurement (GOM ARAMIS)

Images are recorded in a specimen sequence coated with a unique pattern. The images are compared during the measurement, and identical points are searched and tracked. However, tracking a single-pixel through more images is inefficient because a given pixel's color and/or intensity can be similar to the values of more reference image pixels. A possible solution is the permanent identification of smaller areas (so-called facets or subframes - evaluating windows) in the milieu of the pixel in question. The evaluating window is generally a square that can be unmistakably identified through the whole image sequence thanks to the unique pattern preparation of the specimen (Gualtieri, 2012; Freddi, Olmi \& Cristofolini, 2015; GOM, 2021).

Different methods exist to locate an area around a pixel. The two most common methods are normalized cross-correlation and normalized least squares. To reach the required accuracy, the deformation of the evaluating window also needs to be considered; an iteration solution executes it. Most DIC softwares use a variation of the Levenberg-Marquardt algorithm for non-linear optimization (Freddi, Olmi \& Cristofolini, 2015), see Figure 7. 


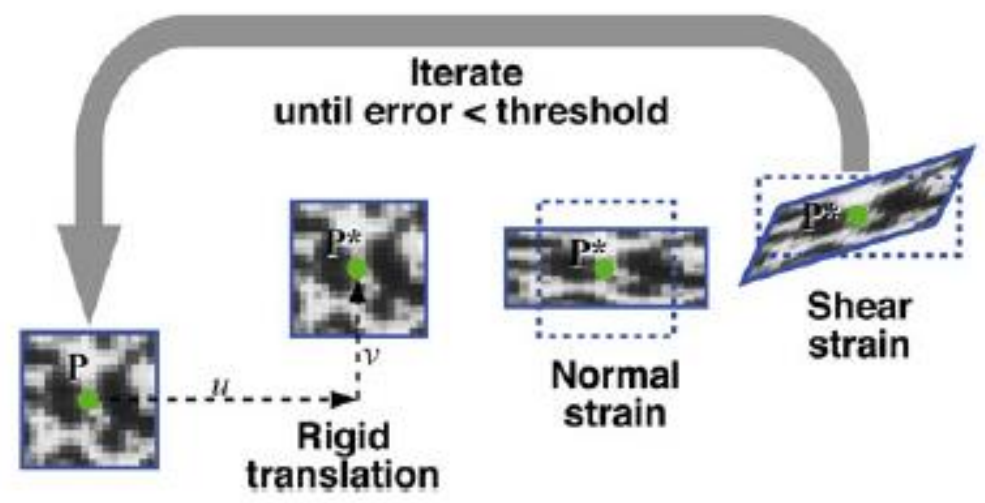

Figure 7. Steps of iteration (Freddi, Olmi \& Cristofolini, 2015)

One of the most critical factors altering the reduction of measurement noise and improving the accuracy of results is the usage of good patterns. GOM systems attach a reference image to the ideal stochastic speckle pattern. When preparing the specimen, the pattern of the actual measurement limit has to be approached as well as possible. The quality of the pattern is essential because we track a small area of the image (evaluating window) while the sample is deforming; then, it is compared to the reference image, attempting to fit the evaluation window permanently as well as possible (Figure 8). This is calculated from the alteration of gray levels and the continuous observation of the identities of the images (Reu, 2014, 2015).
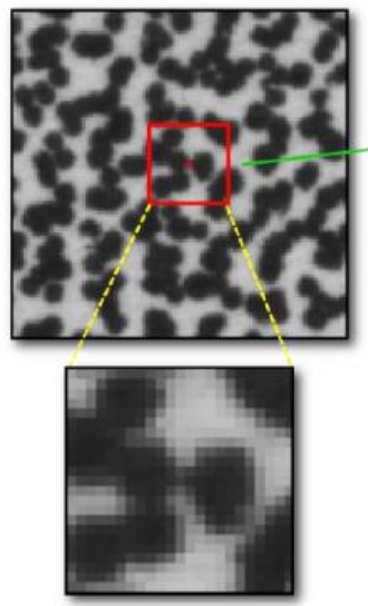
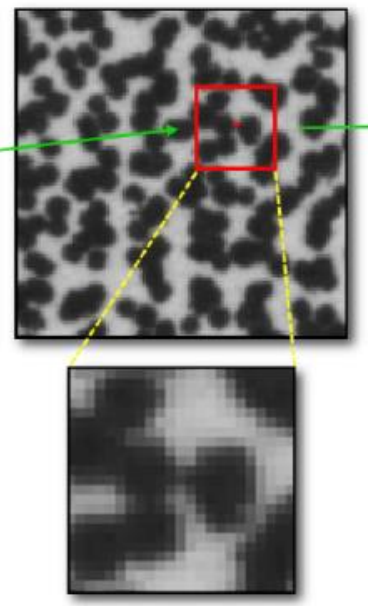
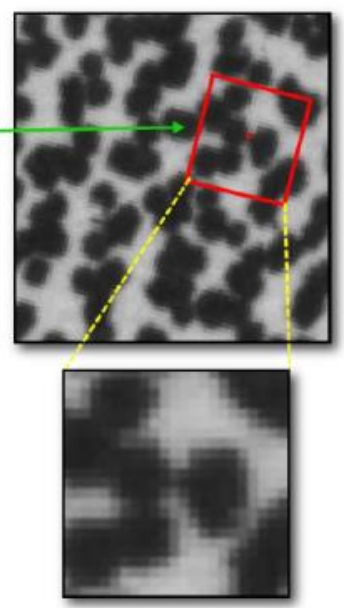

Figure 8. Deformation of the evaluating window during the measurement (Speckle Pattern Fundamentals Correlated Solutions, 2018)

Practical experiences and data from the literature confirm the impact of different effects on the quality of the pattern. The speckle size is calculated by the correlation of the resolution of the camera used for digitization and the area to be measured, still generally, the ideal size of a speckle is between 3-5 pixels. Determination of the contrast is also an essential factor during imaging. White dots can achieve proper contrast on a black basis or black dots on a white basis which significantly helps identification. The best practice is good paint and proper illumination. Aliasing is also a main property of the image. Aliased speckles need to be eliminated in any case since they increase the noise level of the pattern, which endangers the accuracy of the measurement. The density and stochasticity of the speckles are essential requirements as well. Although creating such a uniform pattern that conducts to an incorrect target is rather difficult, the pattern should be as stochastic as possible. Black and white speckles of the same size would be ideal for this reason. Naturally, the white or black basis of the pattern affects the tests, but a white basis with black speckles is more widespread in practice (Burguete et al., 2011). 


\subsection{Validation processes}

Both the systems ATOS and ARAMIS perform contactless measurements, which increases the importance of the calibration process and the validation of the measurement process. The devices of traditional measurement techniques can be used to verify the systems with different methods (Szalai, 2021).

A possible method is the application of the $200 \times 200 \mathrm{~mm}$ specimen of the Nakajima test series used to determine the forming limit curve. The specimen is formed until failure by a hemisphere having $100 \mathrm{~mm}$ as its diameter. To be able to execute the validation feasible for the ARAMIS system too, a proper painted pattern is applied to the sheet. During forming, the deformation of the sheet was tracked by ARAMIS 5M, which enabled the measurement of the changes in thickness.

Thickness reduction of this formed test specimen was measured for the validation process. First, GOM TripleScan II was utilized to digitize the whole surface of the specimen, then the values of thickness decrease were evaluated. Different devices were applied to create the thickness reduction curve along the axis of symmetry of the sample parallelly and perpendicularly to the fracture line (Szalai, 2021):

- a traditional dial indicator (Mitutoyo Absolute Digimatic ID-SX 543-781B),

- an optical microscope (Zeiss Axio Imager M1 with AxioVision 4.8 software and $2.5 \times$ optics),

- a 3D coordinate measuring machine (Mahr PMC 800 coordinate measuring machine with MarSoft Vision 3D 4.1 software).

Thickness reduction is calculated from the surface deformations by ARAMIS, while ATOS measures the sample based on surface digitization. Traditional techniques measure by touching and optical methods. These devices are widely accepted and calibrated, which enables them for the validation of results of GOM systems. The results of the ATOS and the ARAMIS validation measurements can be seen in Table 1 .

Table 1. Results of the ATOS and the ARAMIS validation measurements

\begin{tabular}{|c|c|c|c|c|}
\hline Measuring equipment & $\begin{array}{c}\text { Average thickness next } \\
\text { to the crack } \\
{[\mathrm{mm}]}\end{array}$ & & $\begin{array}{c}\text { Average thickness on } \\
\text { the top } \\
{[\mathrm{mm}]}\end{array}$ & \\
\hline Mitutoyo Absolute Digimatic ID-SX 543-781B & 1.370 & \multirow{3}{*}{1.377} & 1.620 & \multirow{3}{*}{1.631} \\
\hline Mahr PMC 800 coordinate measuring machine & 1.381 & & 1.632 & \\
\hline Zeiss Axio Imager M1 with AxioVision 4.8 & 1.379 & & 1.641 & \\
\hline ATOS TripleScan II & 1.392 & $1.11 \%$ & 1.648 & $1.04 \%$ \\
\hline ARAMIS 5M & 1.414 & $\mathbf{2 . 7 1 \%}$ & 1.644 & $0.80 \%$ \\
\hline
\end{tabular}

During the evaluation of the measurement results, we have to consider the impossibility of measuring on the same points, which is solved by averaging these values. Deviations were $1-3 \%$ in both cases, which is adequate.

Another method was also established to validate the ARAMIS system with a standard tensile test specimen. This method was introduced because the deformation of the painted layer conveys the deformation of the product to the cameras, which is an indirect measurement itself.

Thirteen tensile test specimens were prepared by painting with the following parameters: $20 \mathrm{~mm}$ width, 1.5 $\mathrm{mm}$ thickness, AlMg3 material, gauge length $80 \mathrm{~mm}$. In addition, the specimens' backside was marked by indentations of the Vickers hardness test of $98.1 \mathrm{~N}$ load (HV10) in $50 \mathrm{~mm}$ distances. The accurate values in the initial state were obtained by Zeiss optical length measuring microscope, with $1 \mu \mathrm{m}$ reading accuracy.

The specimens were loaded by Instron 5582 tensile testing machine to $5 \mathrm{~mm}$ units of displacements of the cross-head and $5 \mathrm{~mm} / \mathrm{min}$ velocity. DIC analysis of the specimens was conducted by ARAMIS 5M. After reaching $5 \mathrm{~mm}$ cross-head displacements, the specimens were unloaded. This sequence was repeated until 25 $\mathrm{mm}$ of total displacement (5-10-15-20-25 $\mathrm{mm}$ ). The change of the initial gauge length was defined after each unit of stretching, while the forming phase itself was tracked by ARAMIS. This resulted in 5 cohesive strain increment pairs recorded by DIC technique and traditional length measurement (Szalai, 2021).

After each strain increment, GOM switches its virtual extensometer to a $50 \mathrm{~mm}$ initial value. $\Delta \mathrm{Li}$ increment $[\mathrm{mm}]$ and engineering strain [\%] is calculated compared to this value. Using the traditional length measurement machine, the next increment is always added to the already increased deformation and strain can be calculated this way. The cumulative value of the increments is calculated; thus engineering strains are expressed on the logarithmic scale, since they can be added to each other. The validation test results are presented in Figure 9 (specimen 2) where the equation of the approaching line is in the range of 0.96-1.04 and the calculated average fault is $1.75 \%$. This value is also considered acceptable, thus the validation is successful (Szalai, 2021). 


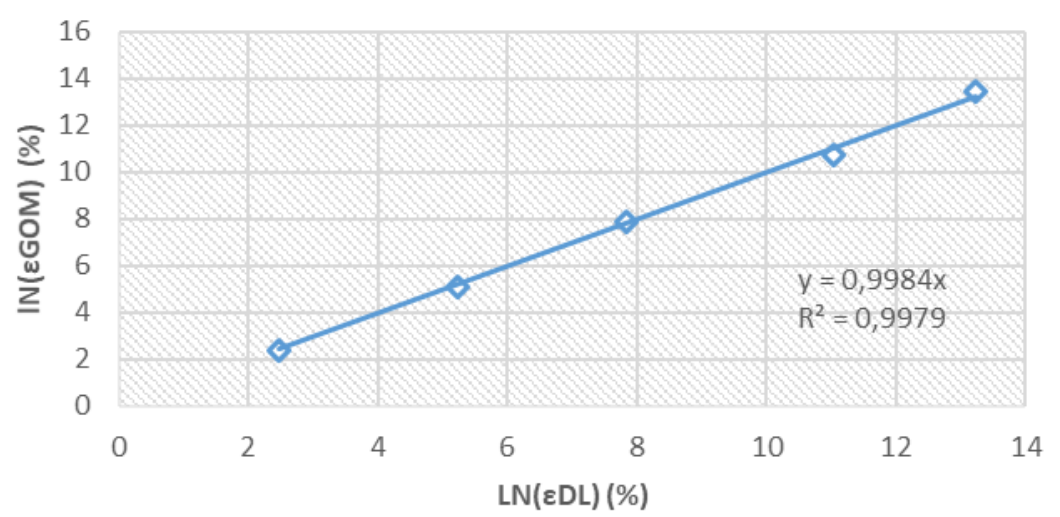

Figure 9. Results of the ARAMIS system validation on sample No. 2. (Szalai \& Dogossy, 2021)

\section{Measurement setup and sample preparation processes}

In the authors' previous publications, they have already measured deformations with the GOM system, focusing on the selection of critical regions of the battery.

In the present study, the advantages, disadvantages, and usability of the two different measurements were examined. Furthermore, in the course of the study, the authors introduced another method, which enables interruption-free analyses. Thus, even dynamic load tests can be executed while continuously monitoring the deformation of the cell.

During the ATOS test, a so-called intermittent test is possible. It means that the battery cannot be measured continuously but only at intervals. However, the measurements will be full geometric measurements, i.e. a full 3D measurement result of the object under study, a point cloud.

The MV170 measuring range and the associated Schneider optics and Titinar projection unit were applied for the study. A measuring distance of $800 \mathrm{~mm}$ is optimal for the measuring range, so the product to be measured must be placed at this distance. For digitization of the entire $360^{\circ}$ surface, the measurement setup was placed and set on a turntable. Before starting the measurement, the system must be calibrated by a calibration sheet according to the measuring range. Reference points are required to combine the images taken at each position. GOM $1.5 \mathrm{~mm}$ high-adhesion coded reference points were applied during the experiments. The battery case was very shiny, so AESUB white scanner spray has been used to reduce reflection (see Figure 10).
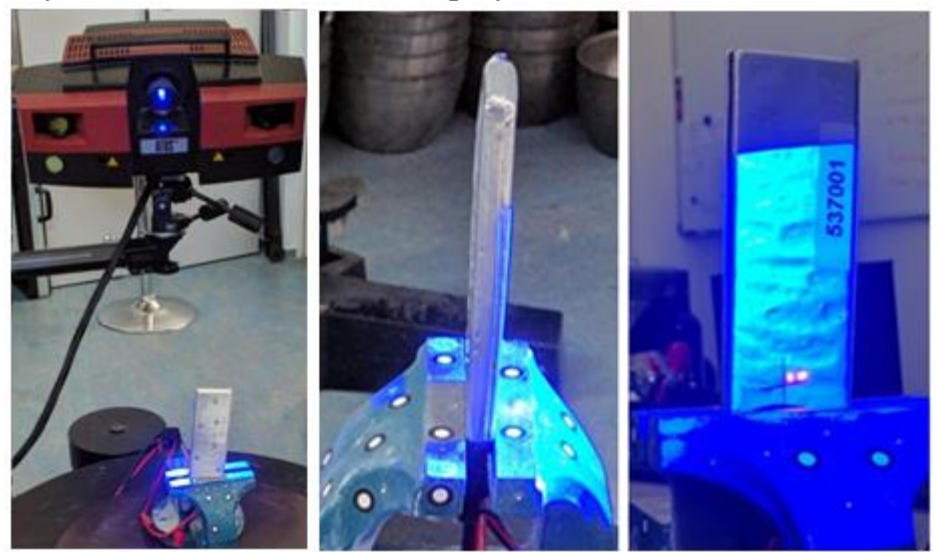

Figure 10. Measuring deformations with GOM ATOS

During the ARAMIS measurements, the entire deformation process was already recorded. Due to the nature of the test, the battery can only be measured from a fixed angle, the cameras cannot be moved during the measurement. The measurement result is also a 3D result with the difference that the product cannot be tested in $360^{\circ}$. The test was performed with an ARAMIS 5M system with an $800 \mathrm{~mm}$ adjustable column, Titinar 50 lenses, and a white LED light source (see Figure 11). The distance between the cameras was $316 \mathrm{~mm}$, the chamber angle was $25^{\circ}$, and the measurement distance was $815 \mathrm{~mm}$. This measurement setting makes it possible to test in a volume of $100 \times 80 \times 50 \mathrm{~mm}$. The system was also calibrated here before starting the measurement. 
The product must be measured with a special paint pattern during the ARAMIS tests, which significantly influences the preparations. Paint has a vital role to play as it transfers deformation. The paint must not dry out too much, but it must be sufficiently dry and secure. Matte white water-based lacquer paint was applied for the test. Here, the painting was completed 12 hours before the measurement, so that proper drying and adhesion can be achieved. A random spot pattern was applied to this layer with a matte black water-based lacquer paint. This layer dried for 60 minutes, which was found to be sufficient and adequate.

The test parameters must be set before starting the measurement. $6 \mathrm{~Hz}$ sampling was used to measure the batteries, so 4500 recordings were prepared of the entire process.
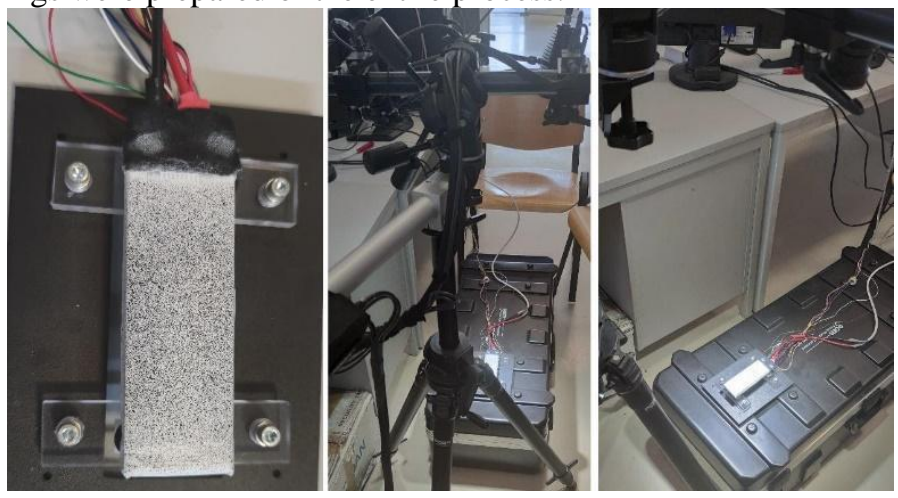

Figure 11. Deformation measurements with GOM ARAMIS system

Electric parameters were required to be recorded to analyze the parameters of the battery. The devices applied in the measurement process have already been presented (Kocsis Szurke \& Lakatos, 2018). The units required to perform the testing processes were as follows: Power supply to charge: Hameg HMP 4030; applied dummy load to discharge: EL-3000; data acquisition to collect voltage data: NI USB 6341; controller software of the test: LabVIEW; the tested lithium polymer battery: Turnigy $5 \mathrm{Ah}$.

The measurements consisted of the following steps in both cases:

- Charging the battery to reach $100 \%$ charging level (SOC).

- Digitize the fully charged state.

- Start loading and pause after a $10 \%$ decrease of energy.

- Conditioning for one hour.

- Digitization of fully discharged state at $0 \%$ SOC.

- Start charging and pause after a $10 \%$ increase of energy.

- In the case of using the ATOS system, digitization was executed in the pauses. This method resulted in images of each $10 \%$ increase and decrease of energy.

- In the case of using the ARAMIS system, the digitization was continuous, but only one side of the battery could be analyzed.

A general image of the process of charging and discharging is presented in Figure 12.
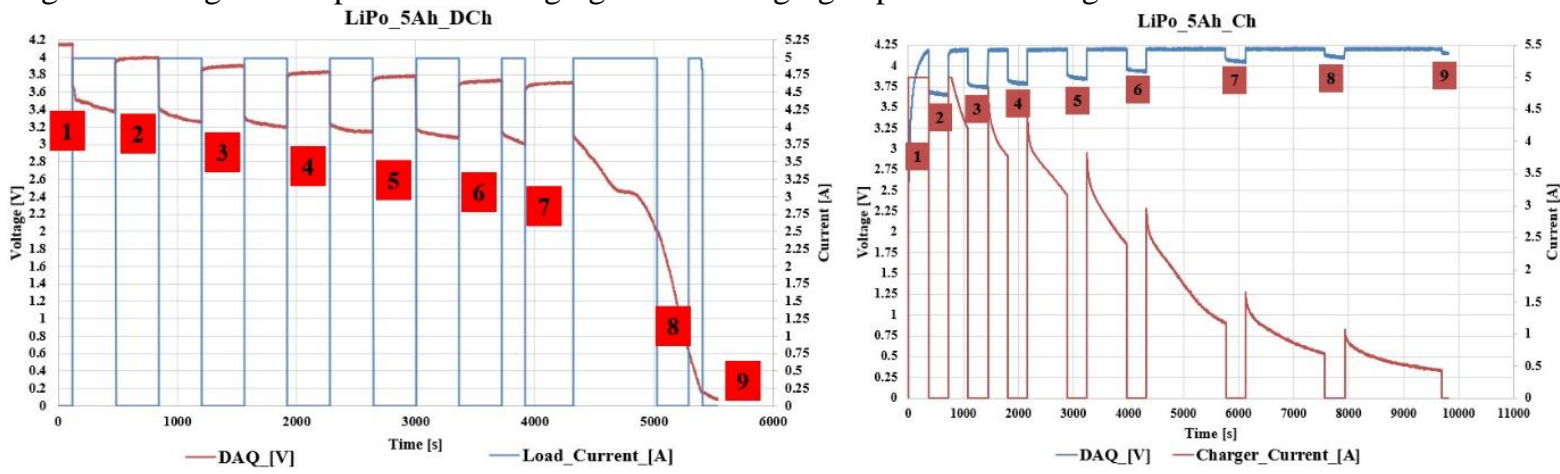

Figure 12. Charging and discharging the lithium polymer battery

On the left side of Figure 12, discharging is shown, voltages marked with red, charging current marked with blue. The discharge rate is $1 \mathrm{C}(5 \mathrm{~A})$ and the voltage limit is $0 \mathrm{~V}$. It is important to note that this kind of discharging destroys the battery; the authors only executed it to reach the maximum deformation. The battery 
charges are presented on the right side of the image; voltage marked with blue, current marked with red. The numbers indicate the digitization points for ATOS measurements at the moments of pausing.

\section{Results and discussion}

Lithium polymer batteries of different conditions were tested. The results are exhibited on a worn-out battery, to demonstrate the highest deformations. Predefined values of charging and discharging have been used for the evaluations. The same cell has been used for the measurements, but it is important to note that higher deformations can appear for the second measurement as a result of the first over-discharging. This research aims to analyze different measurement techniques that are not affected by the battery's condition.

\subsection{Results of charging}

Firstly, the authors analyzed the results of ATOS recorded during charging. A few highlighted images are presented in Figure 13, where the deformation of the lithium polymer battery as a function of the state of charge (SOC) is shown.

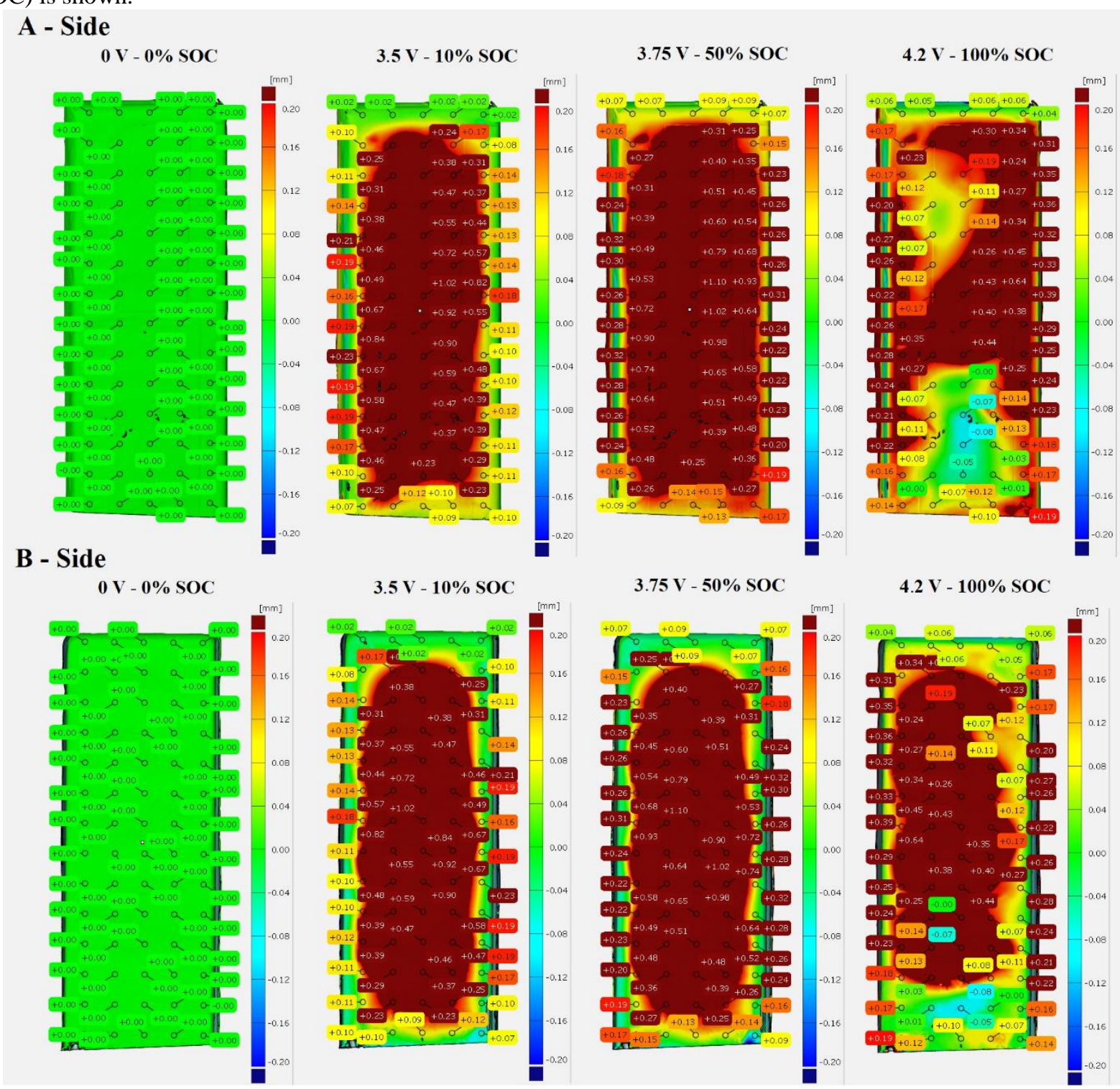

Figure 13. Charging test tracked by ATOS

Since the whole surface of the battery was analyzed during digitization, we own data of both sides of the cell (the lateral sides do not show significant alteration; thus, they can be neglected). Dimensional change of 
side A is presented on the upper half; side B is presented on the lower half of Figure 13. The fully discharged state is shown on the left side of the image (0\% SOC); the second image belongs to $10 \%$ SOC. Such an increase of energy generally does not cause such high deformations, but in the case of a bad condition battery, the deformation starts earlier and faster. This is essential information for vehicle diagnostics because the worn-out battery can be quickly detected by measuring the deformation. In the third image (50\% SOC), side A and B also show further swelling. Surprisingly, contraction can be observed on the right side of the image (fully charged state-100\% SOC) at more locations. The next image summarizes the dispersion of deformations measured at different SOC levels. Deformation values measured at different SOC levels are presented in Figure 14. Deviations originate from the amorphous nature of the cell. The surface of the cell varies in a wide range, the average of the values of the given SOC is represented by AVR.

\section{LiPo battery deformation}

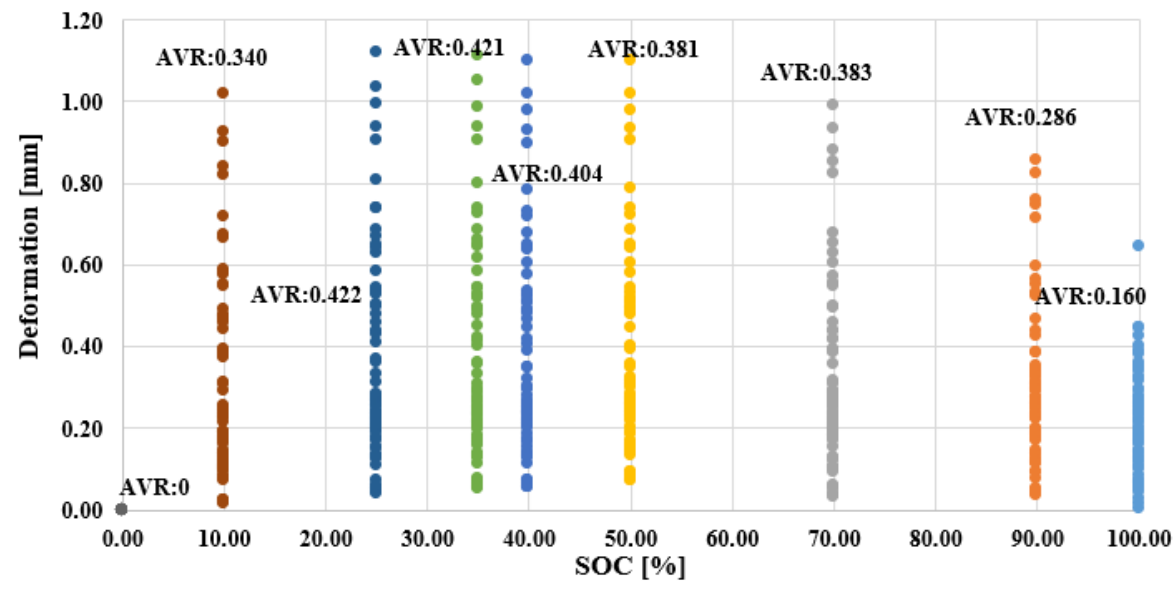

Figure 14. Measuring deviations of deformations by ATOS system at different SOC levels during charging

The ARAMIS measuring system was applied for the second charge condition. The battery rested for $360 \mathrm{~s}$ after a $10 \%$ power charge during the tests. Figure 15 shows some snapshots from the measurements.

$0 \mathrm{~V}-0 \% \mathrm{SOC}$

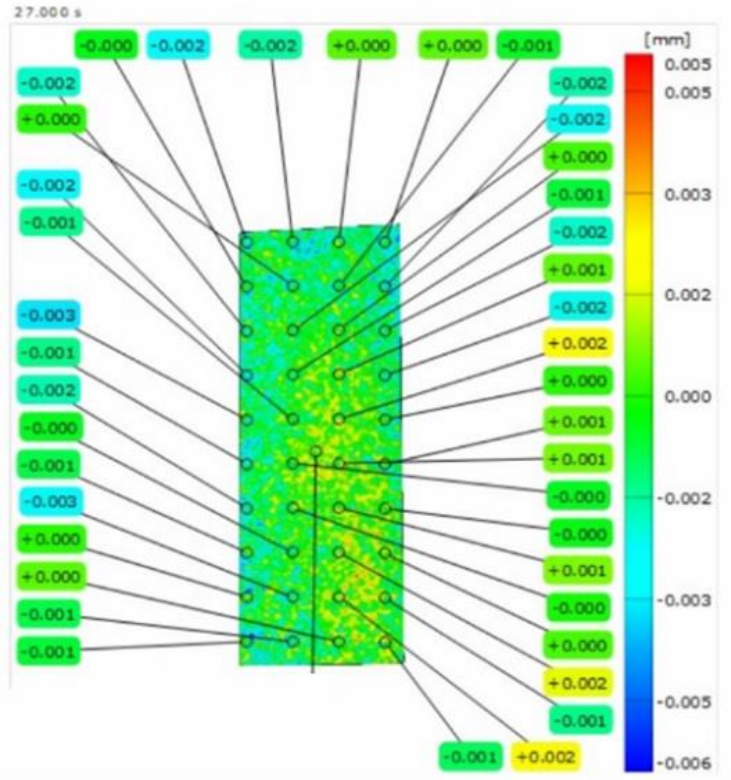

$4.2 \mathrm{~V}-100 \%$ SOC

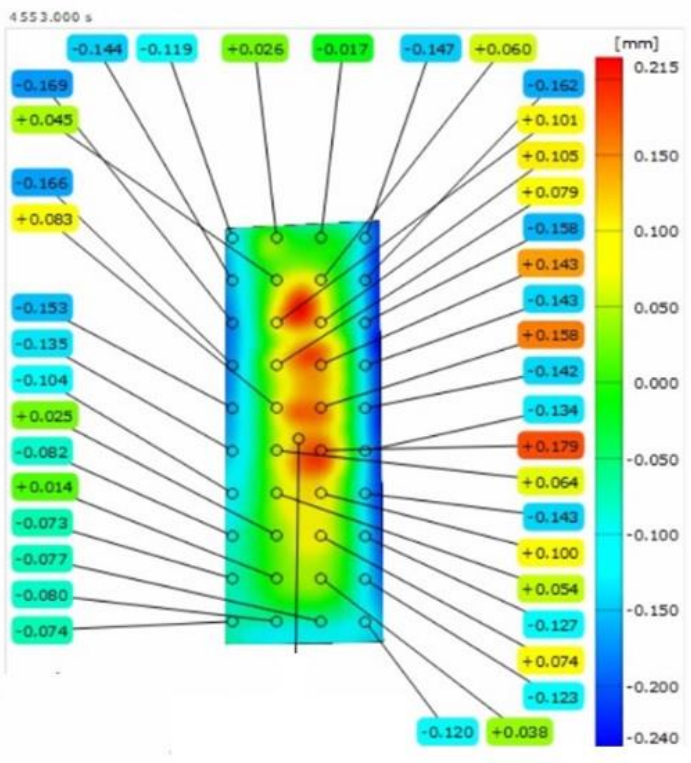

Figure 15. Charging test with ARAMIS measuring system

Measuring points can be determined anywhere on the cell's surface; in this case, they were set in four columns and ten rows, equidistant from each other. The same points were considered and examined during this measurement. (For later analyses, this method can be used to select multiple or possibly other measurement 
locations). When saving the measurement results, it is possible to generate protocols (results) with $1 \mathrm{~s}$ time steps. To be able to guarantee the clarity, only the initial and final states are presented. In Figure 15, the discharged state is observed from the left and the fully charged state is from the right, respectively. Similar to the ATOS measurements, it can be observed that the deformation of the cell shows contraction at some points and humping at other points. Furthermore, it is interesting that a hump was detected in the middle part of the cell and there was a contraction in the outer zones. Figure 16 shows the saved measurement results.

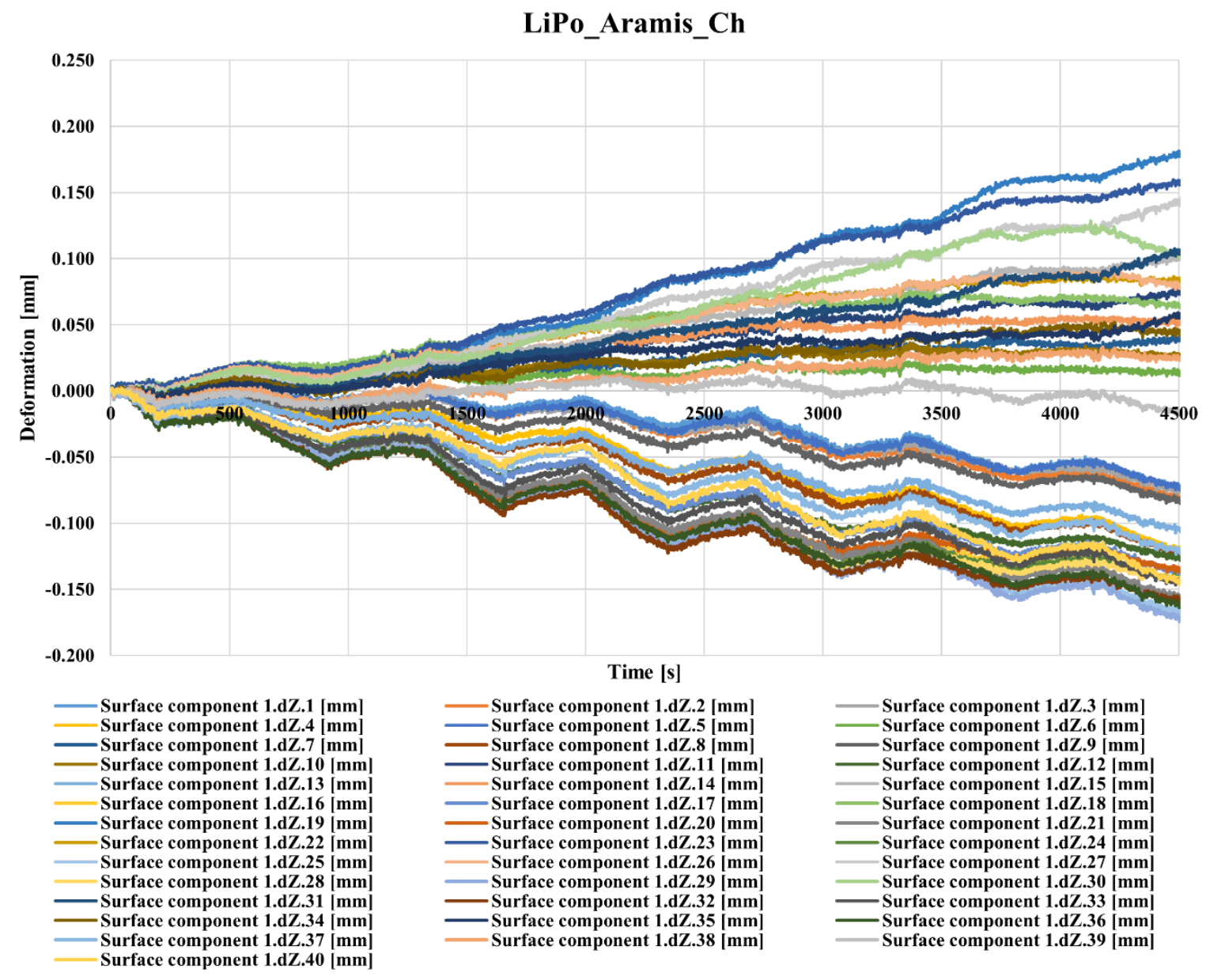

Figure 16. Deformation measured during charging with ARAMIS measuring system

It can be seen in Figure 16 that both contraction and swelling can be detected simultaneously, and it is difficult to determine whether a charge or discharge has taken place by considering the figure alone. Based on this, it can be concluded that the location of the measuring point is an essential factor. It can also be observed from the figure that a periodic ripple occurs during the contraction measurement, which may result from the interruption of the measurements. It is worth studying the phenomenon further to perform further studies with interruptions of different currents and lengths. Another important note is that only one side of the cell can be measured, so the cell as a whole cannot be determined (due to the asymmetric change). 


\subsection{Results of discharging}

The presentation of the discharge results started with the analysis of the values using the ATOS system. Figure 17 shows the deformation recorded during discharge at different charge levels.

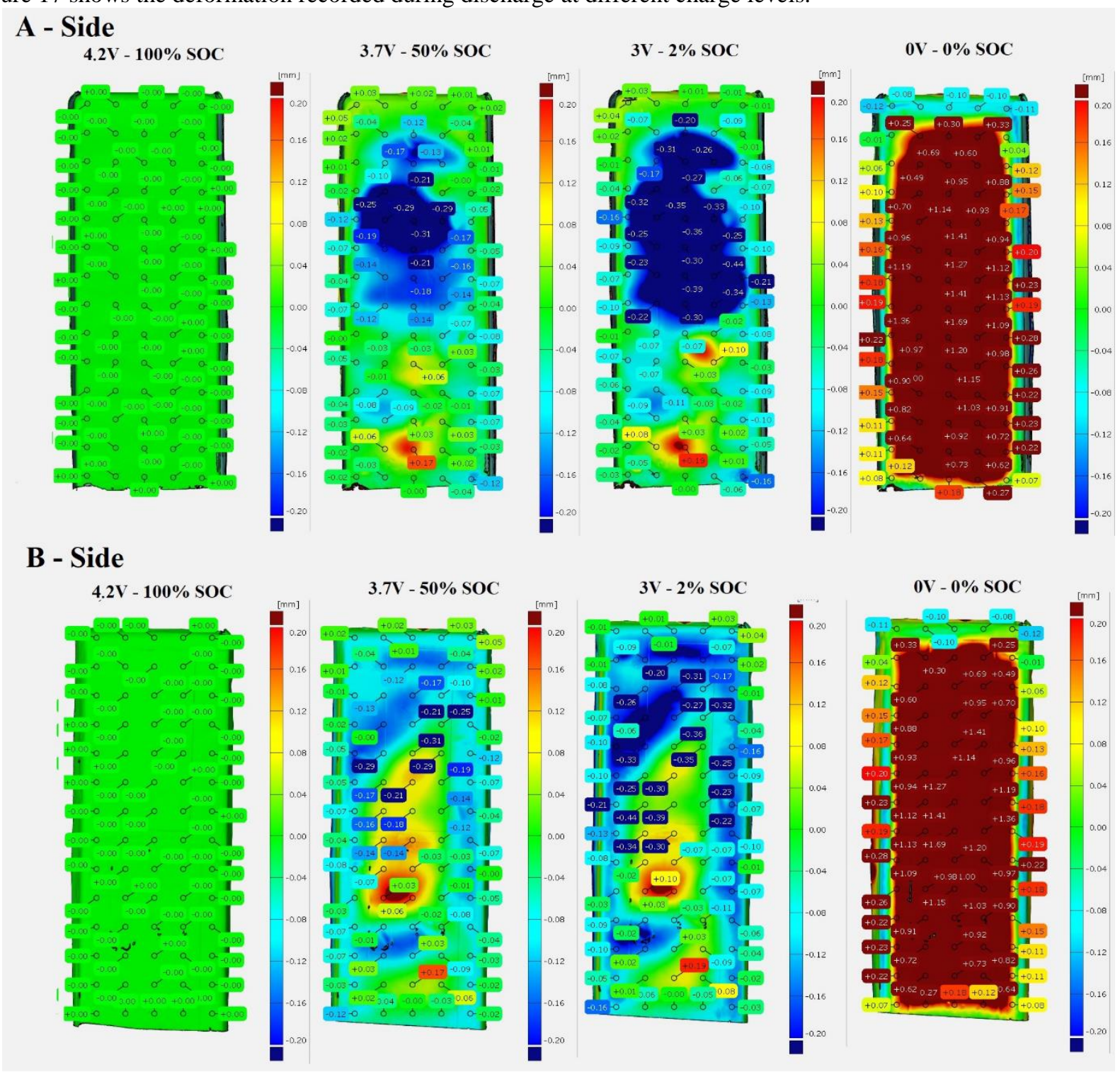

Figure 17. Discharge test using ATOS measuring system

During the measurements, the entire surface of the battery was digitized, so the differences between the respective sides can also be observed (the upper four images are related to side $\mathrm{A}$, the lower four images are related to side B). The fully charged ( $4.2 \mathrm{~V}$ and $100 \%$ SOC) initial state is observed on the left side of the figure. The figure shows a second image of the values recorded at the 50\% charge level. The third image shows the fully discharged state during normal use of $3 \mathrm{~V}$. On the right side of the figure, a state discharged up to 0 $\mathrm{V}$ can be observed, it can be seen that such excessive use is already causing the cell to bump. Figure 18 shows the deformation values obtained for each charge level. 


\section{LiPo battery deformation}

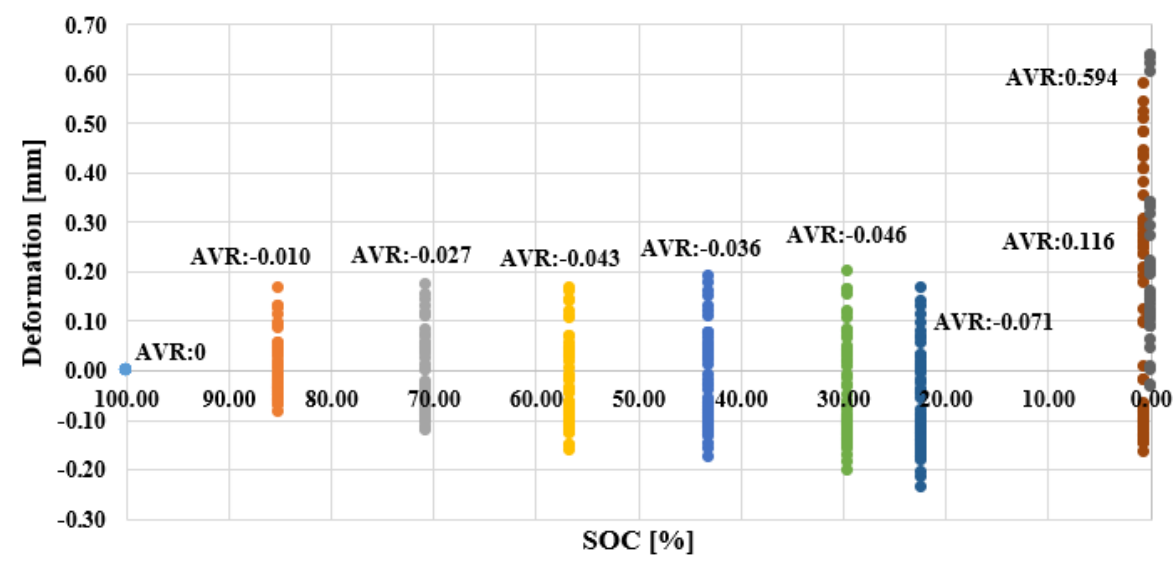

Figure 18. Measuring deviations of deformations by ATOS system at different SOC levels during discharging

Figure 18 shows the deformation data measured at different SOC levels. The differences seen at each charge level (SOC) are due to the amorphous nature of the cell change. It can be seen that the cell surface varies over a relatively large range, with the AVR value being the average of the given SOC in each case.

The second test with ARAMIS was an intermittent discharge test in which sampling was continuous.

The first saved protocol shows a fully charged state (Figure 19 from left). Similar to charging, in this case, a measuring point to any point on the measured side of the cell can be added. The completely discharged state can be seen on the right side of the figure. The rate of change is greater, but the location of change has remained similar. Based on this measurement, it can be observed that the condition of the lithium polymer battery is probably worn-out. As a result, intense humping can be observed in several places during discharge.

\section{$4.2 \mathrm{~V}-100 \% \mathrm{SOC}$}

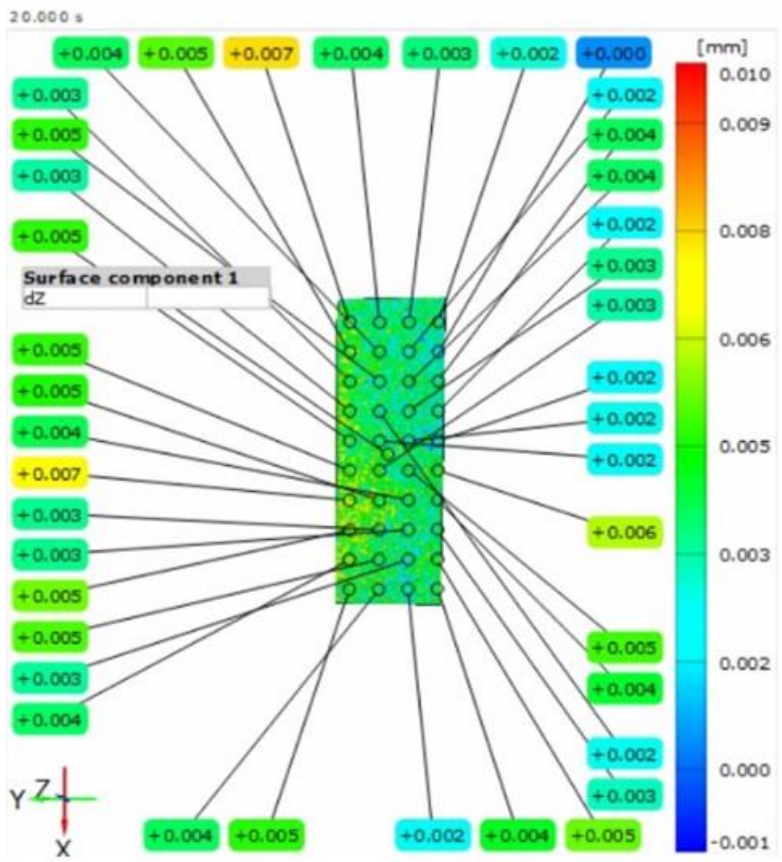

$0 \mathrm{~V}-0 \%$ SOC

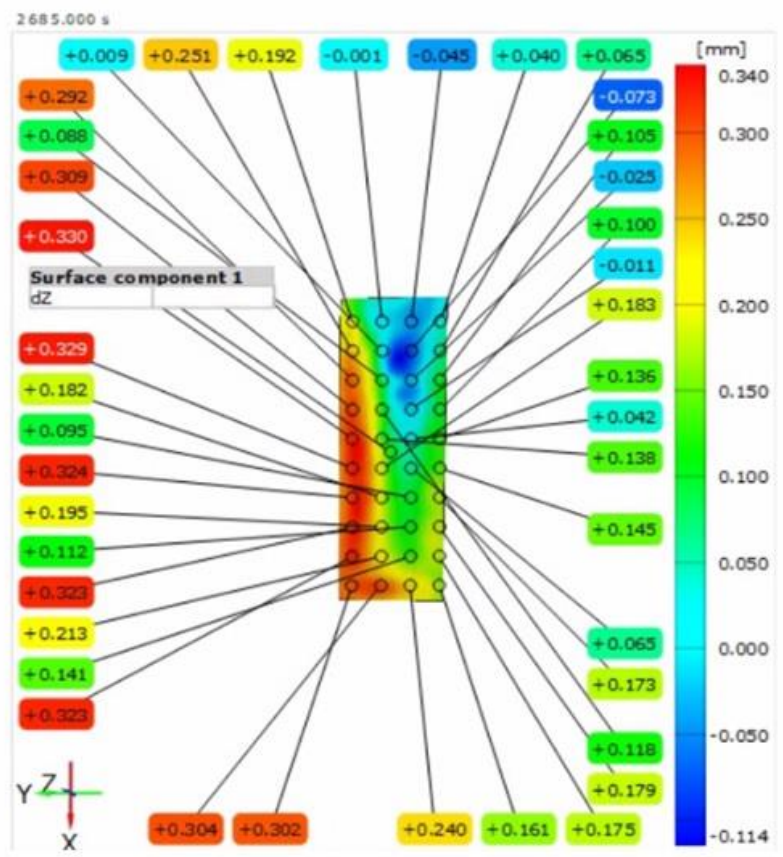

Figure 19. Discharge test with ARAMIS measuring system

The saved deformation data is shown in Figure 20. 


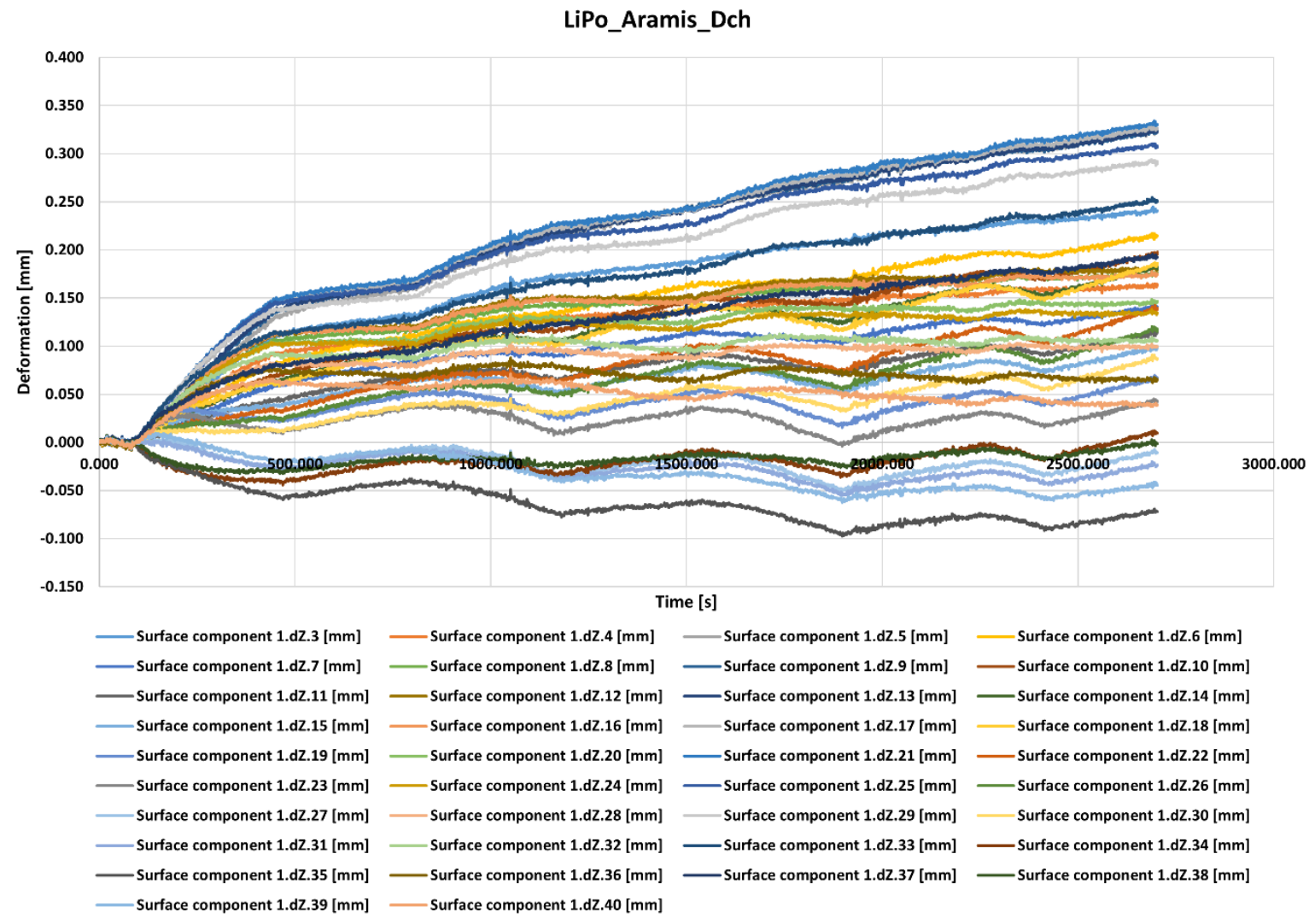

Figure 20. Deformation measured during discharge with ARAMIS measuring system

The development of the bulging (humping) trend based on previous measurements is a significant sign of cell wear. However, substantial additional information in the aspect of battery diagnostics is that there may be a relationship between the degree of cell wear and the area and extent of deformation.

\section{Conclusions}

Both the measurement techniques lead to significant and novel results. The excellent advantage of ATOS is the analysis of the whole surface of the battery. On the other hand, 360 seconds of break is needed to capture the images, which resulted relatively few measured points. Moreover, dynamic tests are not possible to be tracked with this method.

The ARAMIS system allows continuous observation of deformations during the test. The 360 seconds breaks are not required, so dynamic loads are also possible. In contrast, only one side of the battery can be measured, leading to high inaccuracy thanks to the batteries' amorphe and asymmetric behavior. Another disadvantage is that due to the hardware capacity, a maximum of 4500 images can be taken, which is $4500 \mathrm{sec}$ at $1 \mathrm{~Hz}$ resolution. Of course, if the frequency increases, the time decreases. This limit can be widened by developing the IT system. It may be helpful to combine the two methods later:

(i) first, perform a cell condition survey critical region analysis using an ATOS system, i.e., determining a cell asymmetry and compensation value;

(ii) after that, start using the ARAMIS system and examine the cell change in real vehicle measurements.

The disadvantage of the combination is that the two systems do not require the same preparation, and the elimination of asymmetry is also a complex task.

\section{References}

Bajusz, L., Kátai-Urbán, G., Pintér, I., \& Megyesi Z. (2019). Viewpoint correct content projection on arbitrary surface. Gradus, 6(1), 33-42. (in Hungarian) 
Burguete, R. L., Lucas, M., Patterson, E. A., \& Quinn, S. (2011). Speckle pattern characterisation for high resolution digital image correlation. Applied Mechanics and Materials, 70, 261-266. https://doi.org/10.4028/www.scientific.net/AMM.70.261.

Dong, Y. L., \& Pan, B. (2017). A Review of Speckle Pattern Fabrication and Assessment for Digital Image Correlation. Experimental Mechanics, 57(8). https://doi.org/10.1007/s11340-017-0283-1.

Dunn, J. B., Gaines, L., Kelly, J. C., James, C., \& Gallagher, K. G. (2015). The significance of Li-ion batteries in electric vehicle life-cycle energy and emissions and recycling's role in its reduction. Energy and Environmental Science, 8(1), 158-168. https://doi.org/10.1039/c4ee03029j.

Ehrlich, G. M. (2002). Linden's Handbook of Batteries. (4th ed.). New York: McGraw Hill Professional, 2002.

Fotouhi, A., Auger, D. J., Propp, K., Longo, S., \& Wild, M. (2016). A review on electric vehicle battery modelling: From Lithium-ion toward Lithium-Sulphur. Renewable and Sustainable Energy Reviews, 56, 1008-1021. https://doi.org/10.1016/j.rser.2015.12.009.

Freddi, A., Olmi, G., \& Cristofolini, L. (2015). Experimental Stress Analysis for Materials and Structures: Stress Analysis Models for Developing Design Methodologies. Cham: Springer, Springer Series in Solid and Structural Mechanics, 2015. https://doi.org/10.1007/978-3-319-06086-6.

Goda, I., Lhostis, G., \& Guerlain, P. (2019). In-situ non-contact 3D optical deformation measurement of large capacity composite tank based on close-range photogrammetry. Optics and Lasers in Engineering, 119, 37-55. https://doi.org/10.1016/j.optlaseng.2019.02.006.

GOM (2021a). GOM Metrology to https://www.gom.com/en/explore-gom/gom-metrology-to-go (Accessed: 31 December 2021).

GOM (2021b) ATOS Compact Scan.

https://www.gom.com/en/products/3d-scanning/atos-compact-scan (Accessed: 31 December 2021).

GOM GmbH (2011). GOM ATOS III Triple Scan User Manual. https://www.manualslib.com/products/Gom-Atos-Iii-Triple-Scan-10970231.html (Accessed: 31 December 2021).

GOM GmbH (2021). Moules et Outillages de Bourgogne: 3D Scanning of Molds Used for Investment Casting. https://www.gom.com/fileadmin/user_upload/industries/GOM_France_Investment_Casting_Application_Not e_EN.pdf (Accessed: 31 December 2021).

Gualtieri, S. (2012) Novel technique for DIC speckle pattern optimization and generation. PhD thesis, Polotecnico di Milano.

How, D. N. T., Hannan, M. A., Hossain Lipu, M. S., \& Ker, P. J. (2019). State of Charge Estimation for Lithium-Ion Batteries Using Model-Based and Data-Driven Methods: A Review. IEEE Access, 7, 136116136136. https://doi.org/10.1109/ACCESS.2019.2942213.

Jones, E. M. C., Silberstein, M. N., White, S. R., \& Sottos, N. R. (2014). In Situ Measurements of Strains in Composite Battery Electrodes during Electrochemical Cycling. Experimental Mechanics, 54(6), 971-985. https://doi.org/10.1007/s11340-014-9873-3.

Kocsis Szürke, S., Dineva, A., Szalai, S., \& Lakatos, I. (2022). Determination of Critical Deformation Regions of a Lithium Polymer Battery by DIC Measurement and WOWA Filter. Acta Polytechnica Hungarica, 19(2), $113-134$.

Kocsis Szurke, S., \& Lakatos, I. (2018). The lithium polymer battery swelling test with high-precision displacement sensors. 20th International Symposium on Electrical Apparatus and Technologies, SIELA 2018 - Proceedings, 7-10. https://doi.org/10.1109/SIELA.2018.8447119.

Komsiyska, L., Buchberger, T., Diehl, S., Ehrensberger, M., Hanzl, C., Hartmann, C., Hölzle, M., Kleiner, J., Lewerenz, M., Liebhart, B., Schmid, M., Schneider, D., Speer, S., Stöttner, J., Terbrack, C., Hinterberger, M., \& Endisch, C. (2021). Critical Review of Intelligent Battery Systems: Challenges, Implementation, and Potential for Electric Vehicles. Energies 2021, 14(18), 5989; https://doi.org/10.3390/en14185989. 
Leung, P. K., Moreno, C., Masters, I., Hazra, S., Conde, B., Mohamed, M. R., Dashwood, R. J., \& Bhagat, R. (2014). Real-time displacement and strain mappings of lithium-ion batteries using three-dimensional digital image correlation. Journal of Power Sources, 271, 82-86. https://doi.org/10.1016/j.jpowsour.2014.07.184.

Li, J. (2011). A lithium battery pole thickness measurement system based on laser displacement sensor. 2011 International Conference on Electric Information and Control Engineering, ICEICE 2011 - Proceedings, 10161020. https://doi.org/10.1109/ICEICE.2011.5777625.

Li, W., Liu, G., Zhu, L., Li, X., Zhang, Y., \& Shan, S. (2016). Efficient detection and recognition algorithm of reference points in photogrammetry. Optics, Photonics and Digital Technologies for Imaging Applications IV, SPIE Photonics Europe, 2016, Brussels, Belgium, Proceedings 9896, 989612. https://doi.org/10.1117/12.2225416.

Luhmann, T., Robson, S., Kyle, S., \& Boehm, J. (2019). Close-Range Photogrammetry and 3D Imaging, Berlin, Boston: De Gruyter, 2019. https://doi.org/10.1515/9783110607253.

NASA (2014) Short range full-field non-contact structured blue light 3D scanning and photogrammetry for rapid and accurate inspection analysis. https://www.nasa.gov/sites/default/files/files/S_DeRemer-Short_Range_Fullfield_Structured_Blue_Light_3D_Scan_Photo-Gpdf.pdf (Accessed: 31 December 2021).

Popp, H., Koller, M., Jahn, M., \& Bergmann, A. (2020). Mechanical methods for state determination of Lithium-Ion secondary batteries: A review. Journal of Energy Storage, 32, 101859. https://doi.org/10.1016/j.est.2020.101859.

Reu, P. (2012). Introduction to digital image correlation: Best practices and applications. Experimental Techniques, 36(1), 3-4. https://doi.org/10.1111/j.1747-1567.2011.00798.x.

Reu, P. (2014) Speckles and their relationship to the digital camera. Experimental Techniques, 38(4), 1-2 https://doi.org/10.1111/ext.12105.

Reu, P. (2015). Virtual Strain Gage Size Study. Experimental Techniques, 39(5), 1-3. https://doi.org/10.1111/ext.12172.

Rezvanizaniani, S. M., Liu, Z., Chen, Y., \& Lee, J. (2014). Review and recent advances in battery health monitoring and prognostics technologies for electric vehicle ( EV ) safety and mobility. Journal of Power Sources, 256, 110-124. https://doi.org/10.1016/j.jpowsour.2014.01.085.

Rieger, B., Erhard, S. V., Rumpf, K., \& Jossen, A. (2016). A New Method to Model the Thickness Change of a Commercial Pouch Cell during Discharge. Journal of The Electrochemical Society, 163(8), A1566-A1575. https://doi.org/10.1149/2.0441608jes.

Sauerteig, D., Ivanov, S., Reinshagen, H., \& Bund, A. (2017). Reversible and irreversible dilation of lithiumion battery electrodes investigated by in-situ dilatometry. Journal of Power Sources, 342, 939-946. https://doi.org/10.1016/j.jpowsour.2016.12.121.

Slattery, M., Dunn, J., \& Kendall, A. (2021). Transportation of electric vehicle lithium-ion batteries at end-oflife: A literature review. Resources, Conservation \& Recycling, 174, 105755. https://doi.org/10.1016/j.resconrec.2021.105755.

Speckle Pattern Fundamentals Correlated Solutions (2018) Correlated Solutions: Application Note AN-1701. https://www.correlatedsolutions.com/ support/index.php?/Knowledgebase/Article/View/80/1/speckle-patternfundamentals (Accessed: 31 December 2021).

Szalai, S. (2021). Investigation of formability of aluminum vehicle body sheets. PhD thesis, Széchenyi István University (in Hungarian).

Szalai, S., \& Dogossy, G. (2021). Speckle pattern optimization for DIC technologies. Acta Technica Jaurinensis, 14(3), 228-243. https://doi.org/10.14513/actatechjaur.00573.

Tao, R., Zhu, J., Zhang, Y., Song, W. L., Chen, H., \& Fang, D. (2020). Quantifying the 2D anisotropic displacement and strain fields in graphite-based electrode via in situ scanning electron microscopy and digital image correlation. Extreme Mechanics Letters, 35, 100635. https://doi.org/10.1016/j.eml.2020.100635. 
Vagovský, J., Buranský, I., \& Görög, A. (2015). Evaluation of measuring capability of the optical 3D scanner. Procedia Engineering, 100, 1198-1206. https://doi.org/10.1016/j.proeng.2015.01.484.

Wang, Y., Tian, J., Sun, Z., Wang, L., Xu, R., Li, M., \& Chen, Z. (2020). A comprehensive review of battery modeling and state estimation approaches for advanced battery management systems. Renewable and Sustainable Energy Reviews, 131, 110015. https://doi.org/10.1016/j.rser.2020.110015.

Yufit, V., Shearing, P., Hamilton, R. W., Lee, P. D., Wu, M., \& Brandon, N. P. (2011). Investigation of lithiumion polymer battery cell failure using X-ray computed tomography. Electrochemistry Communications, 13(6), 608-610. https://doi.org/10.1016/j.elecom.2011.03.022.

Zhang, N., Tang, H., Zhang, L., \& Trifonova, A. (2015). Asymmetric Electrode for Suppressing Cell Swelling in Commercial Lithium Ion Batteries. Journal of The Electrochemical Society, 162(10), A2152-A2156. https://doi.org/10.1149/2.0881510jes.

Zheng, L., Zhang, L., Zhu, J., Wang, G., \& Jiang, J. (2016). Co-estimation of state-of-charge, capacity and resistance for lithium-ion batteries based on a high-fidelity electrochemical model. Applied Energy, 180, 424434. https://doi.org/10.1016/j.apenergy.2016.08.016.

Zhu, J., Wierzbicki, T., \& Li, W. (2018). A review of safety-focused mechanical modeling of commercial lithium-ion batteries. Journal of Power Sources, 378, 153-168. https://doi.org/10.1016/j.jpowsour.2017.12.034. 